\title{
A NANO INTERDIGITATED ELECTRODES ARRAY ON POLYMER FOR DISPOSABLE IMPEDIMETRIC BIOSENSORS
}

\author{
Zhiwei Zou ${ }^{1}$, Junhai Kai ${ }^{1}$, Michael J. Rust ${ }^{1}$, and Chong H. Ahn ${ }^{1,2}$ \\ Microsystems and BioMEMS Laboratory \\ ${ }^{1}$ Department of Electrical \& Computer Engineering \& Computer Science \\ ${ }^{2}$ Department of Biomedical Engineering \\ University of Cincinnati \\ Cincinnati, OH 45221-0030, USA
}

\begin{abstract}
This paper presents an integrated nano interdigitated electrodes array (IDA) on polymer substrate with microfluidic system. It can be used as a miniaturized, sensitive, and easy-to-use impedimetric sensor for genomics, proteomics, and cellular analysis. A gold $(\mathrm{Au})$ nano IDA has been successfully patterned on polymer (Cyclic olefin copolymer, COC) substrate, which can be widely used for disposable lab-on-a-chip applications. The fabricated device has been characterized in $\mathrm{KCl}$ salt solution using electrochemical impedimetric spectroscopy (EIS). The preliminary result of monitoring protein binding is demonstrated as well, which insures the potential of this device to be used for protein immunosensors.
\end{abstract}

\section{INTRODUCTION AND MOTIVATION}

Electrochemical impedance spectroscopy (EIS) shows detailed information on capacitance and resistance changes from the biorecognition events occurring at the electrode or substrate surface directly, thus, allowing for label-free biosensing [1]. When target DNA hybridizes to oligonucleotide probes or when antibodies bind to antigens, the change in electric properties results in a change of impedance, enabling the measurement of a direct electrical signal. The nanoscale interdigitated electrodes array (nanoIDA) can be introduced to improve the sensitivity since the generated electric field between a gap of $100 \mathrm{~nm}$ matches the region of interest (i.e. on the order of $10-100 \mathrm{~nm}$ for protein immobilization and up to $200 \mathrm{~nm}$ for DNA binding) [2]. In addition, the space confinement of the nanogap minimizes the noise from the electrical double layer [3].

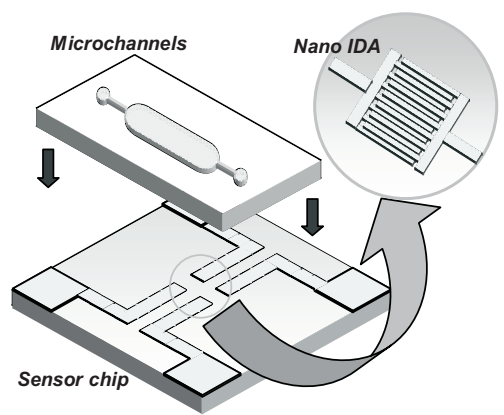

Figure 1. Schematic view of the device including an Au nano IDA on polymer substrate and a polymer microfluidics chip.

High density IDA has been used as a sensing surface to analyze cellular and biomolecular activities in solution using EIS. A small AC voltage is applied to the electrode pairs, thus different concentrations of cells or biomolecules bound to the surface will yield different changes in electrical impedance between the electrodes. This effect has been theoretically analyzed by calculating the electric field between the interdigitated electrodes, and the result is shown and discussed in $[\mathbf{2 , 4 ]}$. The sensitivity of biomolecular analysis has been improved by shrinking the electrodes array size down to the nanoscale due to the electric field being confined much closer to the binding surface. The theoretical calculation indicates that $80 \%$ of the electric field and current flow between two electrodes is in a layer which has thickness less than $L / 2$, where $\mathrm{L}$ is the sum of the electrode width and spacing as shown in Figure 2a. Because the DNA and protein molecular binding layer is less than $200 \mathrm{~nm}$, by applying voltage on one pair of electrodes, most electric field and current flow are limited in this region for nanoscaled electrodes while most parts of the electric field are out of this region for conventional microelectrodes.

Most reported nano IDA structures were fabricated on $\mathrm{Si}$ or glass substrate whereas recently polymer has been considered as one of the most suitable substrate materials for low cost lab-on-achip devices. Patterning metal nano IDA on polymer provide a direct way to integrate the high sensitivity nano biosensor into polymer biochips to utilize both benefits of high sensitivity and low cost. In this work, the nano IDA is patterned on polymer substrates (Cyclic Olefin Copolymer, COC) since COC offers excellent material properties for nano biosensors and is very suitable for disposable polymer lab-on-a-chips [5].
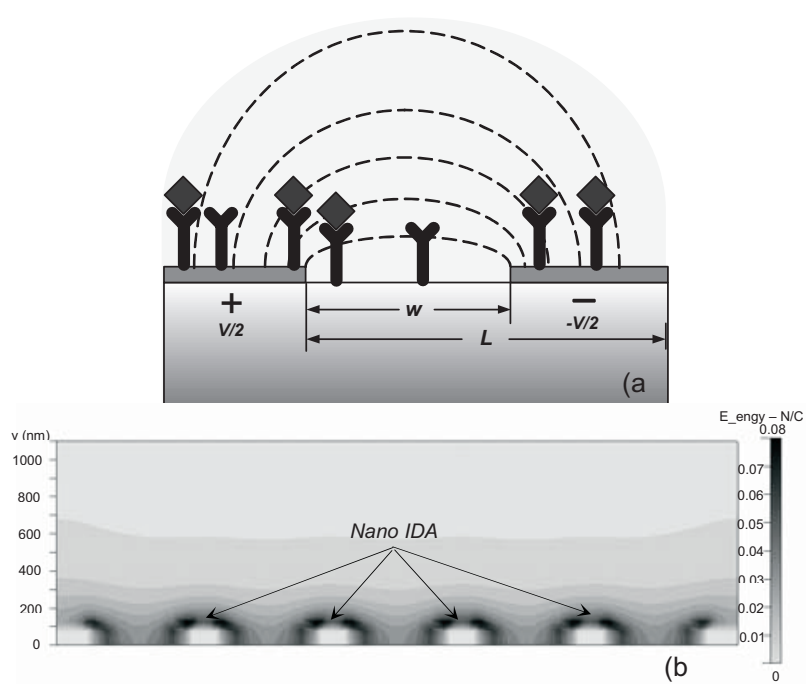

Figure 2. NanoIDA: (a) Design of an Au nano IDA protein binding impedimetric sensor on polymer and (b) FEA simulation of the electrostatic energy distribution of nano IDA in water. $(L=$ $700 \mathrm{~nm}, W=200 \mathrm{~nm}$, and $V= \pm 25 \mathrm{mV}$ ). 


\section{DESIGN AND FABRICATION}

A schematic illustration of the Au nano IDA on polymer with integrated microfluidic system is shown in Figure 1. By using polymer microfluidic chips, the analyte consumption and the total sensing time are significantly reduced.

FEA simulation is used to analyze the electric field energy around the Au nanoIDA in water. Three pairs of Au electrodes are modeled with $100 \mathrm{~nm}$ height, $200 \mathrm{~nm}$ width, and $500 \mathrm{~nm}$ spacing in water medium as shown in Figure 2. The simulation result clearly indicates that all the electric filed is concentrated within $600 \mathrm{~nm}$ of the sensor surface where protein binding occurs.

The fabrication processes is summarized in Figure 3. A 3-inch blank COC wafer with very good surface smoothness is prepared by plastic injection molding techniques using a highly polished, mirror face Ni mold-disk. A Cr layer of $10 \mathrm{~nm}$ is first evaporated on the COC to make the substrate compatible with e-beam lithography. Electron resist Polymethylmethacrylate (PMMA) is spin-coated and the pre-baking condition is adjusted due to the relatively low melting point of COC. E-beam lithography is performed by Raith-150 system, exposing the PMMA. The nano pattern is defined after developing and $\mathrm{Cr}$ etching. Subsequently, a Au layer of $100 \mathrm{~nm}$ is evaporated on the patterned substrate by ebeam metal evaporator. The sample is then placed in acetone for lift-off; the $\mathrm{Au}$ nanoIDA is created in this step. After nanofabrication, photolithography techniques are used for nanomicro interface patterning and integration of $\mathrm{Au}$ nanoIDA with electrical connection pads. A second Au layer $(200 \mathrm{~nm})$ is evaporated, covering the existing contact pads achieved by the nano fabrication. The sample is then dipped in acetone for lift-off. Finally, all the redundant $\mathrm{Cr}$ is etched out.
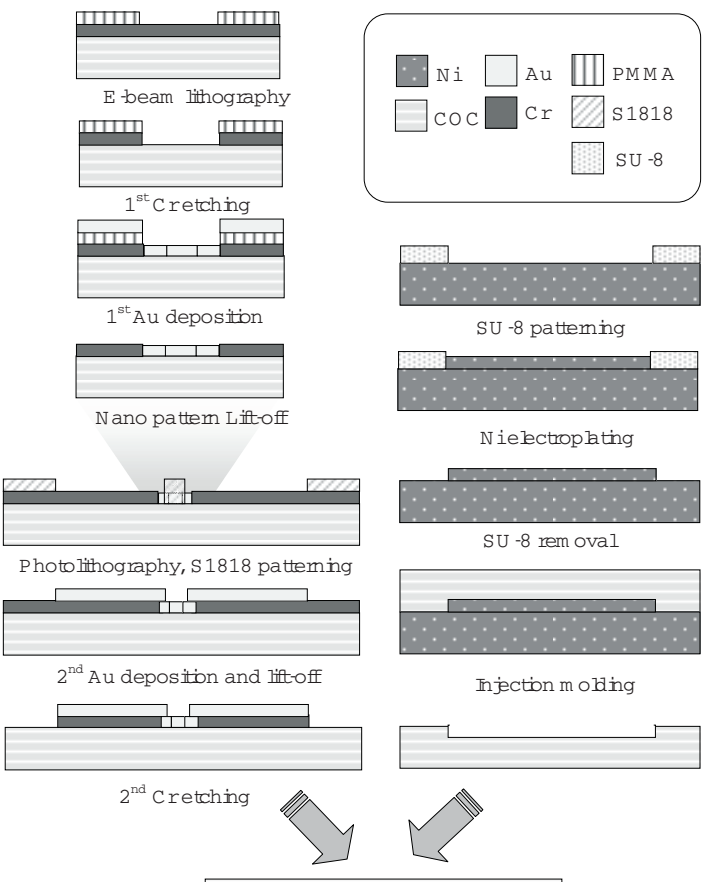

SU -8 patteming

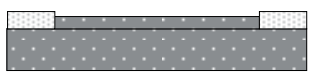

Nielectrophting

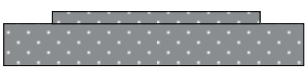

SU -8 rem oval

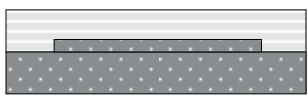

Injection m olaing
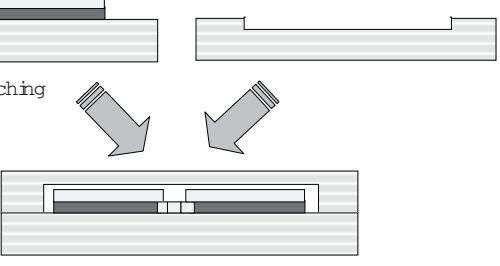

Therm albonding
In order to fabricate the polymer microfluidic system, the plastic injection molding and thermal bonding techniques have been developed [6]. The SU-8 2035 photoresist is spin-coated on the 3 -inch nickel disk to achieve 100 um thickness. Then, a prebake process is performed. After the photoresist layer is exposed with UV source, it is baked again for the cross-linking. After developing the SU-8 photoresist, the electroplating is performed in a nickel plating bath, using a two-electrode system with a nickel anode and a nickel disk cathode with SU-8 patterns. Finally, a nickel mold with a plating microstructure is attained after removal of the residual SU-8 photoresist. The microfluidic chip was replicated from this mold over a plastic substrate of COC by injection molding. After drilling holes for fluidic interconnection at inlets and outlets, the microfluidic chip is bonded with the sensor chip using thermal fusion bonding technique to make the final device.

Photographs of the fabricated device are shown in Figure 4. Figure $4 \mathrm{a}$ and $4 \mathrm{~b}$ illustrate the configuration of $\mathrm{Au}$ nanoIDA, electrical connections and microchannel. The images of nanofabricated IDAs on polymer by SEM and AFM are shown in Figure $4 \mathrm{c}, 4 \mathrm{~d}$, and $4 \mathrm{e}$. The nanoIDA consists of 100 electrode fingers with $100 \mu \mathrm{m}$ length, $200 \mathrm{~nm}$ width, $100 \mathrm{~nm}$ thickness and $500 \mathrm{~nm}$ spacing in the $0.01 \mathrm{~mm} 2$ sensing area.
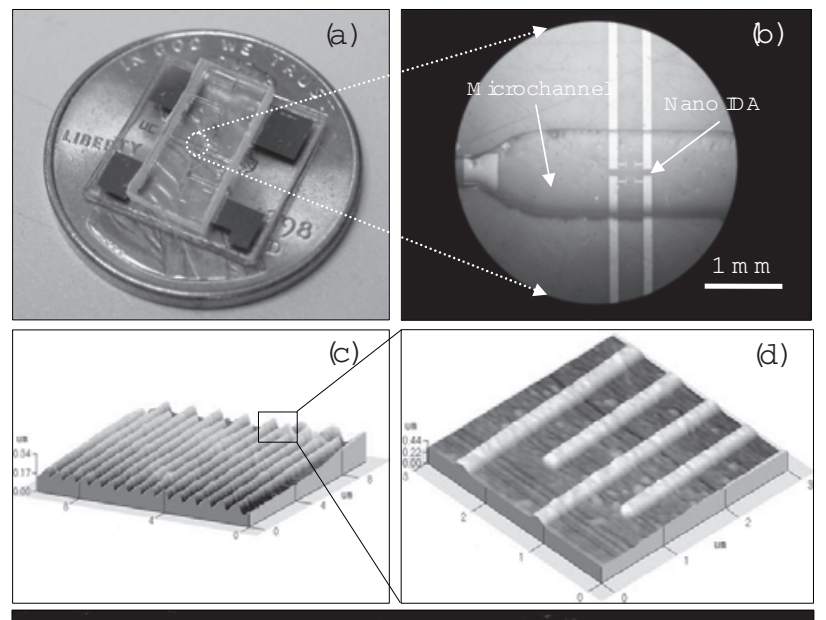

(d)

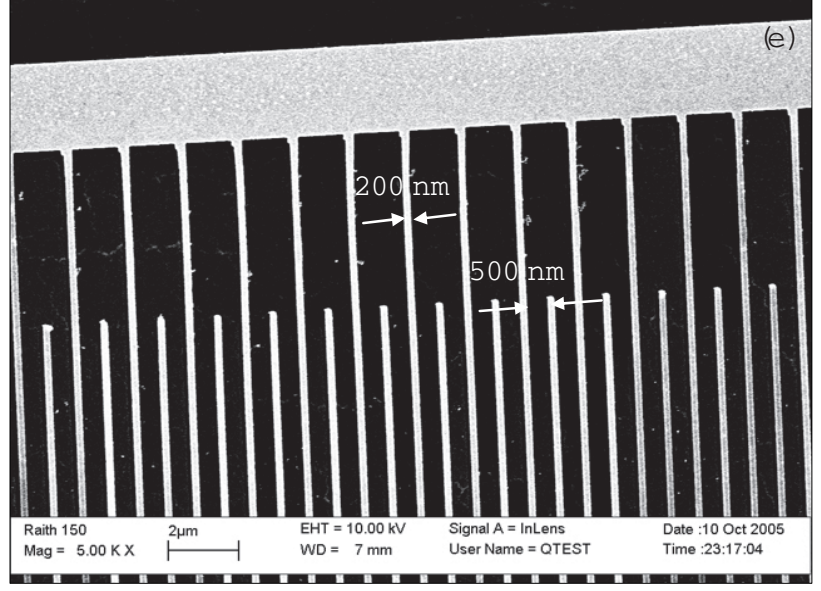

Figure 4. Photographs of the nanodevice: (a) the entire chip; (b) microscope image of nano IDA, electrical connections and microchannel; (c), (d) AFM; and (e) SEM images of the nano IDA array on polymer substrate.

Figure 3. Fabrication processing for the nanoIDA with polymer microfluidic channels. 


\section{RESULTS AND DISCUSSION}

The nano device is electrically characterized in $\mathrm{KCl}$ solution with various concentrations using HP 4284A LCR meter (Figure 5). The EIS is performed at $50 \mathrm{mV}$ with the frequency range $20 \mathrm{~Hz}$ to $1 \mathrm{MHz}$ after the sample solution is injected into the sensing channel. The DI water and $\mathrm{KCl}$ solutions are measured from low to high concentration using the same method. By analyzing a typical impedance response of the nano IDA in $10^{-3} \mathrm{M} \mathrm{KCl}$ solution, three regions can be subdivided as shown in Figure 6a.
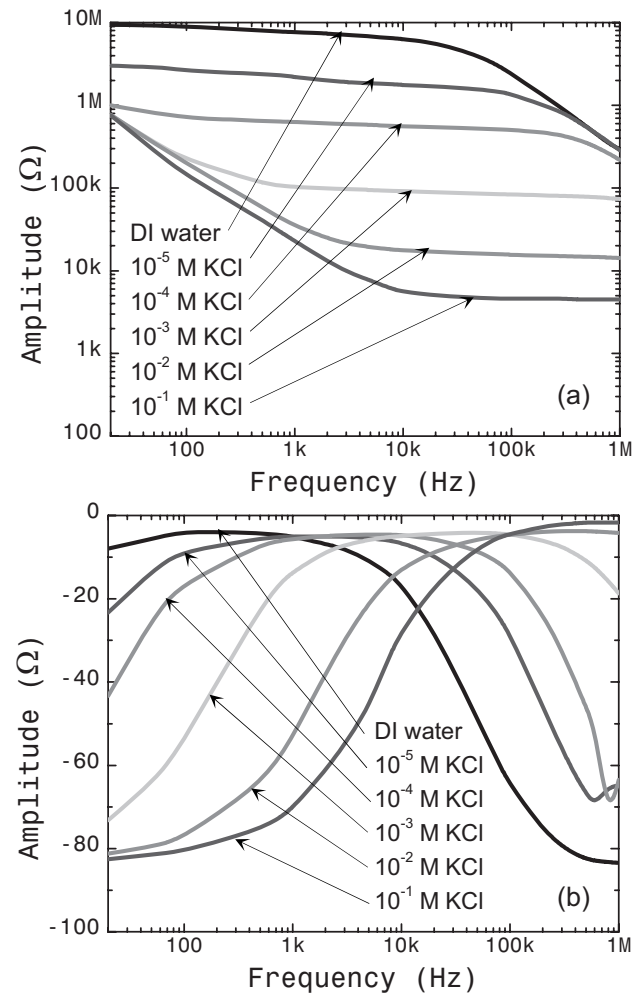

Figure 5. EIS of a nanoIDA under $\mathrm{KCl}$ electrolyte with different concentrations: a) amplitude response and (b) phase response.

A simplified electrical model [2] (Figure 6a) can be used to explain these three regions. They correspond to three major electrical components: the serial double layer capacitance $C_{d l}$, the serial electrolyte resistance $R_{\text {sol }}$, and the parallel solution capacitance $C_{d i}$. At the low frequency range, $C_{d l}$ determines the signal. Phenomena occurring in the neighborhood of the electrode are measured in this region, thus surface related processes influence this capacitance. At intermediate frequencies, $R_{\text {sol }}$ governs the signal, and the conduction of ions in the solution determines the signal. At the high frequency end, the dielectric behavior can be observed. The dielectric behavior of solution $C_{d i}$ dominates the signal in this region. Further measurement and analysis of this simplified model is presented in Figure 6 . In the range from $20 \mathrm{~Hz}$ to $100 \mathrm{KHz}$, the capacitance of $10^{-2} \mathrm{M} \mathrm{KCl}$ solution $C_{d i}$ can be neglected. The agreement of the fitting curve coming from the model and the measurement data is shown in Figure 6.
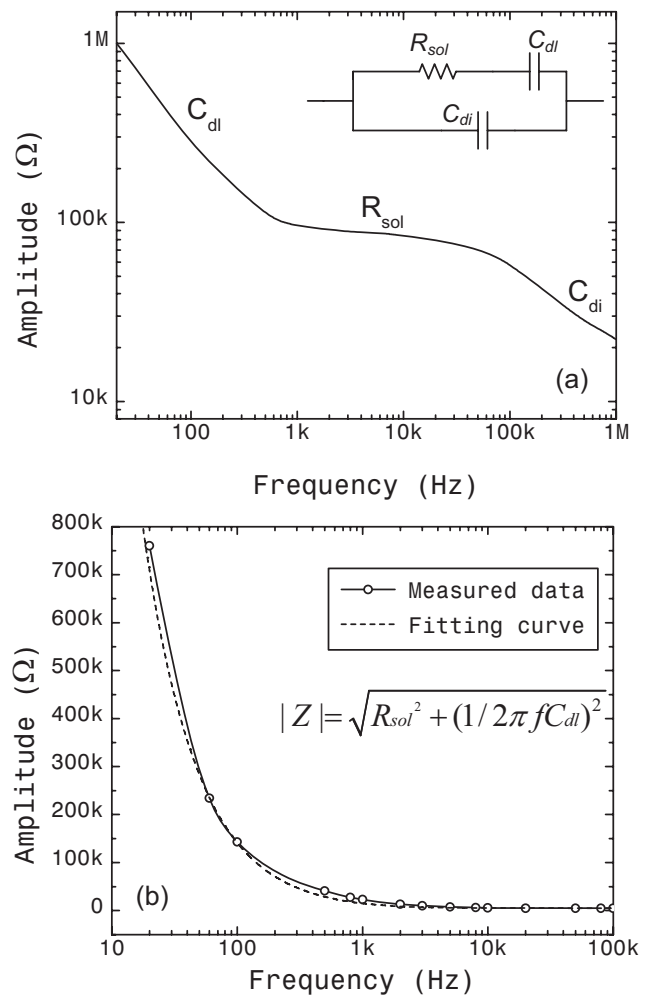

Figure 6. Electrical modeling for the nanoIDA in the electrolyte: (a) impedance amplitude spectrum in the $10^{-3} \mathrm{M} \mathrm{KCl}$ solution and the three different impedimetric response regions which can be explained by the (inset) simplified electrical model and (b) Impedimetric response in a $10^{-2} \mathrm{M} \mathrm{KCl}$ solution in the frequency range from $20 \mathrm{~Hz}$ to $100 \mathrm{KHz}$. The fitting curve coming from the simplified electrical model is also given with $C_{d l}=15 \mathrm{nF}$ and $R_{\text {sol }}=$ $5 K \Omega$.

One of possible applications for an integrated microchannel/EIS system is the investigation into the dielectric properties and bio-affinity interaction of proteins, which includes the protein binding to functionalized electrode surface and the antibody-antigen specific binding. These interactions create a new charged layer as a capacitance that is serial to the $C_{d l}$; hence decrease the $C_{d l}$ and increasing the impedance at the low frequency range. This analysis has been verified by the preliminary test. Figure 7 gives the result of impedance change when proteins (Mouse monoclonal anti-rabbit immunoglobulin, mouse anti-rabbit $\operatorname{IgG})$ are immobilized. The immobilization of proteins is based on the formation of based self-assembled monolayer (SAM) of alkanethiols group on Au electrodes surface. The sensor chip is preconditioned in a 11-Mercaptounadecanoic acid (MUA) and 3-Mercaptopropionic acid (MPA) mixing solution and activated with 1-(3-Dimethylaminopropyl)-3ethylcarbodiimide (EDAC) and N-hydroxysuccinimide (NHS) before testing [7]. The sample solutions with different concentrations of mouse anti-rabbit $\mathrm{IgG}$ are injected into the sensing microchannel and held for 5 minutes for incubation. After completely washing out the samples, the EIS is performed in the same PBS buffer solution. As shown in Figure 7a, impedance change occurs at the low frequency range in which $C_{d l}$ dominates as we expected. Accordingly, the relative impedance increase at 
$100 \mathrm{~Hz}$ with varying protein concentrations are recorded (Figure $7 b)$.
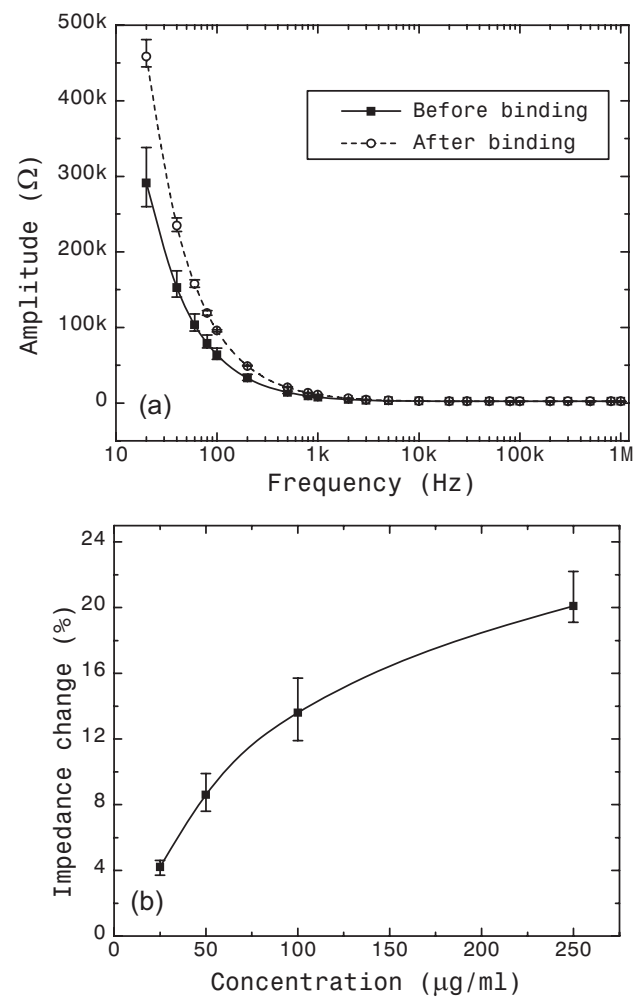

Figure 7. (a) Impedimetric response: (a) Before and after protein binding $(0.25 \mathrm{mg} / \mathrm{ml}$ mouse anti-rabbit $\operatorname{IgG})$ and (b) Calibration curve of relative impedance change versus different mouse antirabbit IgG concentrations at $100 \mathrm{~Hz}$.

As proof of concept, this preliminary results show a potential of the device as an immunosensor with high sensitivity. Further investigation is under progress to improve the sensor's performance such as the sensitivity and specificity. Specificity of the target protein could be achieved by utilizing the antibodyantigen specific binding and Bovine serum albumin (BSA) surface pre-blocking. Moreover, $\mathrm{COC}$ shows the natural property to absorb protein molecule by hydrophobic force for an excellent specific binding [8]. Thus, future investigation can be employed to monitor the impedance change caused by the COC surface absorbed protein between the electrodes pair, which would greatly shorten the sensor preparation by eliminating the SAM formation step.

\section{CONCLUSIONS}

In this work, a novel nanoIDA on polymer has been nanofabricated and successfully characterized as EIS biosensors. Using $\mathrm{KCl}$ solution, the measured impedance spectra has shown a good matching to the theoretically predicted impedance spectra. Preliminary testing results for the EIS nano biosensor indicate that this nano biosensor has a high potential for rapid, direct, and labelfree biomolecular and cellular analysis. In addition, the nanosensor can be easily integrated with disposable polymer lab-on-a-chip.

\section{ACKNOWLEGEMENTS}

The authors gratefully thank Mr. Ron Flenniken, Mr. Robert Jones, and Mr. Jeff Simkins in the Institute of Nanotechnology at the University of Cincinnati for their technical support. This work has been partially supported from NSF-P921-L856 and NIH-P021L684. The Raith e-beam lithography system is supported from NSF Grant \#0216374.

\section{REFERENCES}

[1] E. Katz and I. Willner, "Probing Biomolecular Interactions at Conductive and Semiconductive Surfaces by Impedance Spectroscopy Routes to Impedimetric Immunosensors, DNASensors, and Enzyme Biosensors", Electroanalysis, 15, 11 (2003), pp. $913-947$.

[2] P. Van Gerwen, W. Laureyn, W. Laureys, G. Huyberechts, M. O. D. Beeck, K. Baert, J. Suls, W. Sansen, P. Jacobs, L. Hermans, and R. Mertens, "Nanoscaled Interdigitated Electrodes Array for Biochemical Sensors", Sensors and Actuators B, 49 (1998), pp. 73 - 80.

[3] M. Yi, K. H. Jeong, and L. P. Lee, "Theoretical and Experimental Study towards a Nanogap Dielectric Biosensor", Biosensor and Bioelectronics, 20 (2005), pp. 1320 - 1326.

[4] M. W. den Otter, "Approximate Expressions for the Capacitance and Electrostatic Potential of Interdigitated Electrodes", Sensor and Actuators A, 96 (2002), pp. 140 - 144.

[5] C. H. Ahn, J. W. Choi, G. Beaucage, J. H. Nevin, J. B. Lee, A. Puntambekar, and J, Y. Lee, "Disposable Smart Lab on a Chip for Point-of-Care Clinical Diagnostics", Proceedings of the IEEE, 92 (2004), pp. 154 - 173.

[6] J. W. Choi, S. Kim, R. Trichur, H. J. Cho, A. Puntambekar, R. L. Cole, J. R. Simkins, S. Murugesan, K. S. Kim, J. B. Lee, G. Beaucage, J. H. Nevin, and C. H. Ahn, "A Plastic Micro Injection Molding Technique Using Replaceable Mold-Disks for Disposable Microfluidic Systems and Biochips", Proceedings of the 5th International Conference on Micro Total Analysis Systems (microTAS 2001), Monterey, CA, Oct. 21 - 25, (2001), pp. 411 - 412.

[7] M. Veish, Y. Zhang, K. Hinkley, and M. Zhang, "TwoDimensional Protein Micropatterning for Sensor Applications through Chemical Selectivity Technique," Biomedical Microdevices, 3 (2001), pp. 45 - 51.

[8] J. Kai, Y. S. Sohn and C. H. Ahn, "Protein Microarray on Cyclic Olefin Copolymer (COC) for Disposable Protein Lab-on-aChip," Proceedings of the 7th International Conference on Micro Total Analysis Systems (micro-TAS 2003), Squaw Valley, CA, Oct. 5-9, (2003), pp.1101-1104. 